\title{
Analysis of the experimental data measured by BM@N drift chambers
}

\author{
Ján Fedorišin ${ }^{1,2, *}$ \\ ${ }^{1}$ Veksler and Baldin Laboratory of High Energy Physics, Joint Institute for Nuclear Research, 141980 \\ Dubna, Moscow region, Russia \\ ${ }^{2}$ Institute of Experimental Physics SAS, Watsonova 47, 04001 Košice, Slovakia
}

\begin{abstract}
The current status of the analysis of the experimental data measured by the drift chambers in the BM@N experiment is presented. Apart from the previously published analysis based on the deuteron beam data, this study shows preliminary results for the carbon data at energy $4.5 \mathrm{GeV}$ per nucleon. Some practical issues, such as evaluation and possible removal of the combinatorial background, as well as reliability of the employed track reconstruction algorithm are discussed as well.
\end{abstract}

\section{Introduction}

BM@N (Baryonic Matter at Nuclotron) [1] is the first experiment at the JINR NICANuclotron complex. The main aim of BM@N is to study multistrange hyperons and light hyper-nuclei produced in the interactions of relativistic heavy ion beams with fixed targets at kinetic energies varying from 1 to $6 \mathrm{GeV}$ per nucleon. The elaborated description of the BM@N set-up and the experimental program are presented in [1,2] and [3].

Two identical drift chambers DCH1 and DCH2 [4] of the outer tracker are located outside the magnetic field of SP41 analyzing dipole magnet [5]. Each DCH is composed of four segments measuring different track coordinates in a transverse (xy) plane relative to the beam axis. The measured coordinates are $x, y, u, v$ where $u$ and $v$ are Cartesian coordinates rotated by angles $\pm 45^{\circ}$ around $z$ axis.

\section{$2 \mathrm{DCH}$ track reconstruction}

The primary experimental variable directly measured by drift chambers is the electron drift time. The typical drift time spectrum for the BM@N carbon beam data at $E_{k i n}=4.5 \mathrm{AGeV}$ is shown in Fig. 1 on the left.

To convert these drift times to the distances of the closest approach (DCA) of tracks to the anode wires, a radius-time calibration curve must be constructed. Providing the DCA distribution is to be uniform, the calibration curve is estimated by integration of the drift time spectrum

$$
r(t)=\frac{r_{\max }}{N_{\mathrm{tot}}} \int_{t_{0}}^{t} \frac{d N}{d t^{\prime}} d t^{\prime},
$$

\footnotetext{
*e-mail: fedorisin@jinr.ru
} 

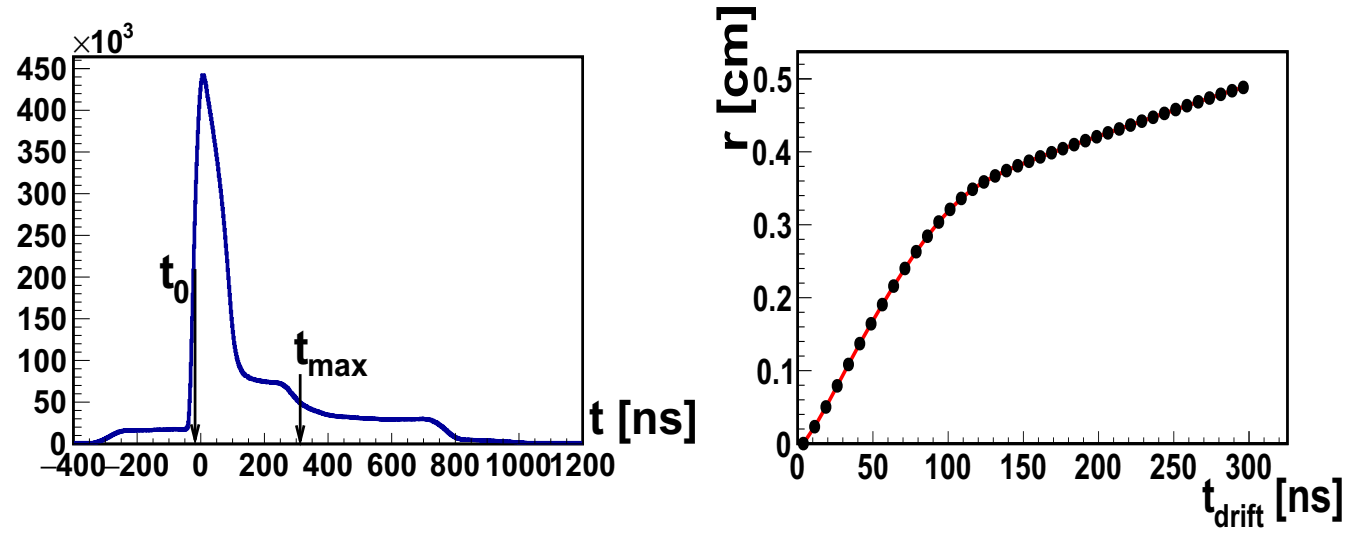

Figure 1. Drift time spectrum for the carbon beam data (on the left) and the corresponding $r$ - $t$ calibration curve (on the right)

where $N_{\text {tot }}$ is the total number of DCH hits and time $t$ ' gradually increases in equidistant steps from $t_{0}$ to $t_{\max }$. The time limits $t_{0}$ and $t_{\max }$ correspond to the minimal and the maximal drift distances, respectively, i.e. to $r_{0}=0$ and $r_{\max }=0.5 \mathrm{~cm}$. Fig. 1(right) shows the $r$ - $t$ calibration curve estimated for the drift time spectrum in Fig. 1(left).

The DCA coordinates are then used to calculate one of the $x, y, u, v$ coordinates in the corresponding DCH's planes and the missing $x$ or $y$ coordinates are evaluated by solving a set of linear equations. Finally, after correcting the track candidates for misalignment of the drift chambers, the track combinations from both the drift chambers are fitted by straight lines to produce global DCH track candidates. The outlined track reconstruction procedure is iteratively repeated until the averaged track residual spectra are minimized.

\section{A few remarks on the analyzed data}

Fig. 2 displays the profile of the carbon beam in the first plane of the first DCH. The empty gap visible on the right side of the reconstructed beam corresponds to the DCH central aperture.

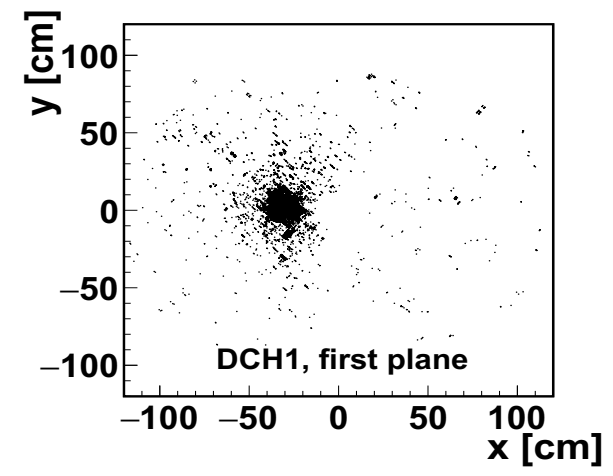

Figure 2. Profile of the carbon beam at $E_{k i n}=4.5 \mathrm{AGeV}$ 
Since this histogram is made at the early stages of the track reconstruction process, there is still a lot of background scattered all over the picture. This behaviour of the background indicates a poor quality of the measured experimental data. Indeed, this conclusion is supported by the spectrum presented in Fig. 3 on the left which shows a number of DCH's hits per event for the same data.
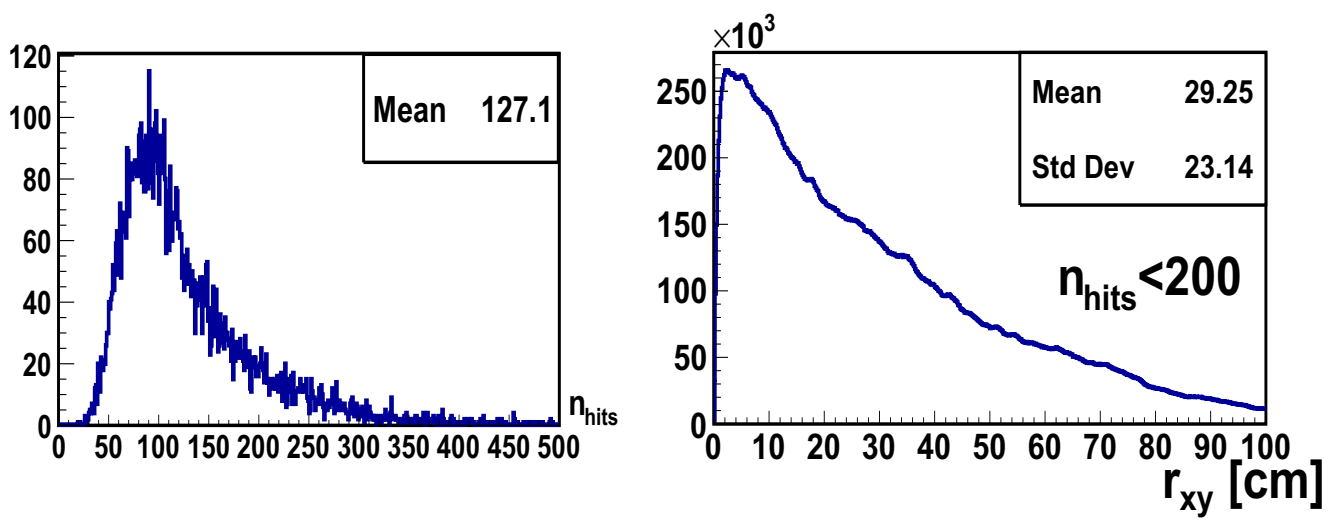

Figure 3. Number of DCH's hits per event (on the left) and distance $r_{x y}=\sqrt{x^{2}+y^{2}}$ of track hits from two consecutive DCH planes (on the right). The second spectrum is shown for the preselected events with DCH's hit multiplicities under 200

Since each DCH consists of 8 anode wire planes, for an ideal case the beam should leave no more than 16 hits in both the drift chambers. However, as one can see in Fig. 3, the mean number of DCH's hits is much higher which implies that most of the detected hits are just undesired background.

The situation gets even worse after combining the hits from different DCH planes when constructing the track candidates. The abundant occurrence of false hits in DCH planes leads to a huge combinatorial background and consequently to the unproportional increase of fake track candidates. This is demonstrated in Fig. 3 on the right that shows distance $r_{x y}=\sqrt{x^{2}+y^{2}}$ of track hits from two consecutive DCH planes, i.e. the transverse plane projection of the full distance. It is estimated that due to the small carbon beam deflection angles (a few degrees) this distance should be less than $2 \mathrm{~cm}$, therefore all the entries in the presented plot with $r_{x y} \gtrsim 2 \mathrm{~cm}$ are fake track candidates and must be discarded.

The opportunities to reduce the background levels in the experimental data and at various stages of the track reconstruction procedure are presently investigated and evaluated.

\section{Verification test of the track reconstruction algorithm}

The results presented in this section are only preliminary and the track reconstruction algorithm is still worked on to be further improved.

Quite a simple check of the DCH track reconstruction algorithm is based on the momentum reconstruction of the carbon beam. If the beam with initial momentum $p=p_{z}$ moves in a homogeneous magnetic field, it is deflected in $x z$ plane by angle $\varphi_{x z}[6]$ :

$$
\varphi_{x z}(\operatorname{rad})=q \frac{\int B d l}{p},
$$


where $B$ is $B_{y}$ component of the magnetic flux density, $q$ is a beam particle charge and $\int B d l$ is the integral of magnetic field along the beam path.

Thus, if the experimental value of the beam deflection angle is estimated, the reconstructed beam momentum is given by the following expression:

$$
p=q \frac{\int B d l}{\varphi_{x z}} .
$$

Fig. 4 shows the dependences of reconstructed deflection angle $\varphi_{x z}$ and beam momentum $p$ on the magnetic field integral. The different values of $\varphi_{x z}$ and $\int B d l$ have been obtained
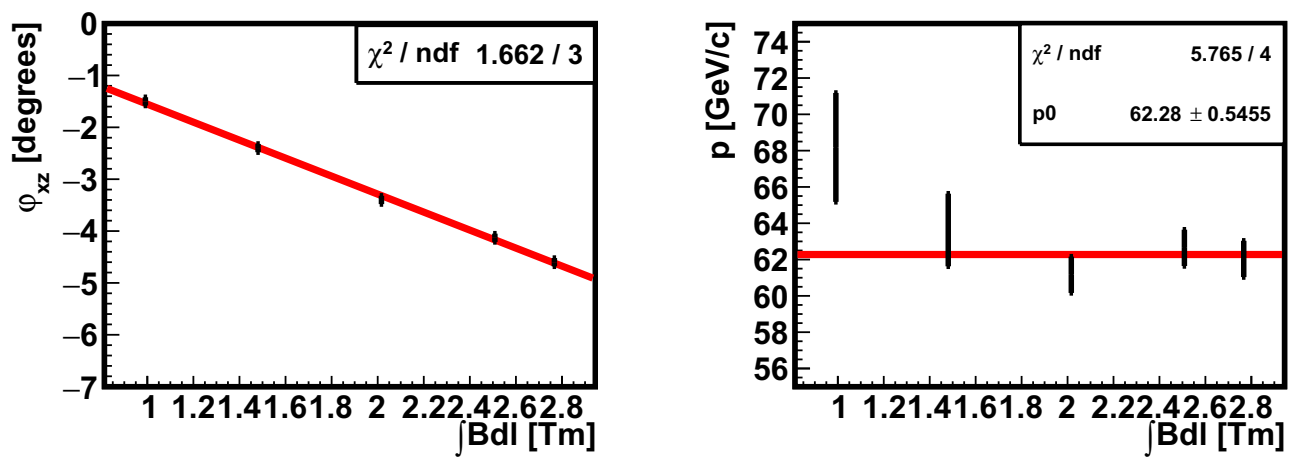

Figure 4. Reconstructed deflection angle of the carbon beam in $x z$ plane (on the left) and reconstructed carbon beam momentum (on the right) as functions of magnetic field integral

from a couple of experimental runs with the different magnetic flux densities $B$. The linear behaviour observed in the first plot and the constantness of the beam momentum indicated in the second plot are in agreement with the functions (2) and (3), respectively. The final value of the reconstructed carbon beam momentum estimated as constant fit of $p$ vs $\int B d l$ dependence is thus $62.3 \pm 0.5 \mathrm{GeV} / \mathrm{c}$ which is more than three statistical errors lower than nominal value $64.21 \mathrm{GeV} / \mathrm{c}$. This indicates that there is still some source of systematic errors that must be found and eliminated from the analysis.

\section{Summary}

1. The complex algorithm of the particle hit and track reconstruction in the drift chambers has been tested on the BM@N carbon beam data.

2. The applied algorithm includes the estimation of the $r$ - $t$ calibration curve, hit finding and formation of the local and global DCH track candidates.

3. The track candidates are corrected for the DCH's geometric misalignment and inaccuracies of the $r$ - $t$ calibration curve.

4. The momentum of the carbon beam is reconstructed in order to test reliability of the applied method.

5. Although the preliminary results seem to be promising, some work still has to be done, above all to make up an efficient algorithm capable to reduce the excessive background observed in the studied experimental data. The geometric alignment procedure requires further improvements as well. 


\section{References}

[1] T.O.Ablyazimov et al. (BM@N Collaboration), BM@N-Baryonic Matter at Nuclotron (Conceptual Design Report), http://nica.jinr.ru/files/BM@N/BMN_CDR.pdf (2012)

[2] M. Kapishin, Eur. Phys. J. A 52, 213 (2016)

[3] M. Kapishin at al. (BM@ N collaboration), Eur. Phys. J. A 52, 182 (2018)

[4] D. Béderéde et al., Nucl. Instrum. Meth. A 367, 88-91 (1995)

[5] P.G. Akishin et al., physics.ins-det arXiv:1407.7096v1 (2014)

[6] Tables of Physical $\mathcal{E}$ Chemical Constants (16th edition 1995) (Kaye \& Laby Online. Version 1.0, 2005) http://www.kayelaby.npl.co.uk 